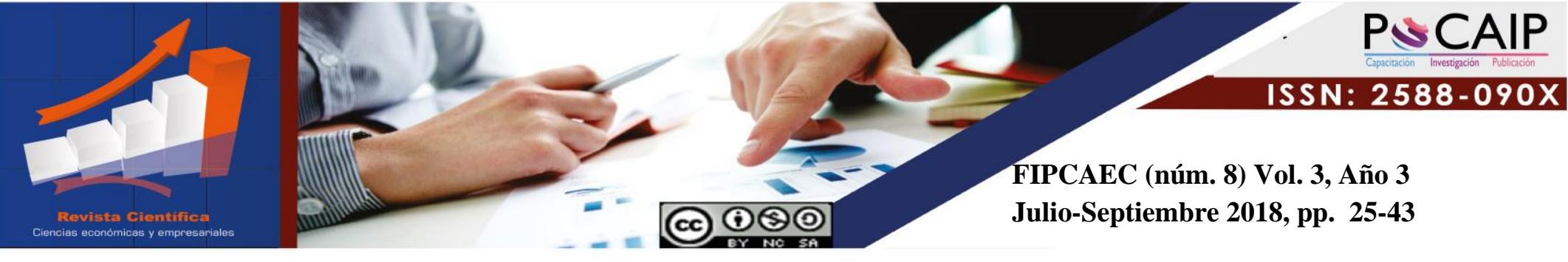

DOI: DOI 10.23857/fipcaec.v3i8.56

\title{
Las empresas su organización y funcionamiento
}

\section{The companies its organization and operation}

\section{As empresas sua organização e operação}

\author{
Lenin Párraga-Zambrano ${ }^{1}$ \\ lenin_andres_p@hotmail.com \\ https://orcid.org/0000-0002-2070-6217
}

Correspondencia: lenin_andres_p@hotmail.com

* Recepción: 21/ 04/ 2018 * Aceptación: 12/06/ 2018 *Publicación: 15 /07/ 2018

1 Doctor en Ciencias Administrativas, Magíster en Gerencia Educativa, Diploma Superior en Liderazgo Educativo, Ingeniero Comercial, Docente de la Universidad Laica Eloy Alfaro de Manabí, Extensión Chone, Chone, Ecuador. 


\title{
Resumen
}

En la actualidad las empresas están afrontando procesos complicados y dinámicos, en los que demandan el apoyo del administrador. Entre estos procesos, destaca por su importancia la necesidad de implementar estrategias con las que puedan generar ventajas competitivas, que les garanticen enfrentar con mayores posibilidades de éxito los retos y oportunidades en un entorno cada vez más competitivo. Se realiza una revisión bibliografía acerca de la temática, donde se abordan aspectos como, la definición de administración, sus funciones, gerencia estratégica.

Palabras clave: Administración; empresa; estrategias; productividad.

\begin{abstract}
At present, companies are facing complicated and dynamic processes, in which they demand the support of the administrator. Among these processes, the need to implement strategies with which they can generate competitive advantages, which guarantee them to face challenges and opportunities in an increasingly competitive environment with greater possibilities of success, stands out for their importance. A bibliography review is carried out on the subject, where aspects such as administration definition, functions, strategic management are addressed.
\end{abstract}

Keywords: Administration; company; strategies; productivity.

\section{Resumo}

Atualmente, as empresas enfrentam processos complicados e dinâmicos, nos quais exigem o apoio do administrador. Dentre esses processos, destaca-se a importância de implementar estratégias com as quais possam gerar vantagens competitivas, que garantam enfrentar desafios e oportunidades em um ambiente cada vez mais competitivo e com maiores possibilidades de sucesso. Uma revisão bibliográfica é realizada sobre o assunto, onde são abordados aspectos como definição de administração, funções e gestão estratégica.

Palavras-chave: Administração; empresa; estratégias; produtividade 


\section{Introducción}

La administración es el resultado histórico e integrado de la contribución acumulada de diversos precursores, filósofos, físicos, economistas, estadistas y empresarios que, con el paso del tiempo, fueron desarrollando y divulgando, cada uno, obras y teorías en su campo de actividades. Por tanto, la a administración moderna utiliza conceptos y principios empleados en las Ciencias Matemáticas (incluso la estadística), en las Ciencias Humanas (como Psicología, Sociología, Biología ,Educación, etc.), en las Ciencias Físicas, así como en Derecho, Ingeniería, Tecnología de la informatización, etc. Es importante afirmar que la administración se ha practicado durante mucho tiempo, esfuerzos realizados por aquellas personas responsables de planear, organizar, dirigir y controlar actividades han existido desde que existe a humanidad. (Introducción a la administración. 2018)

\section{Atenciones de la administración en las organizaciones}

En la actualidad la Administración es reverenciada por sus contribuciones al proceso de desarrollo de la humanidad como un fenómeno universal; se le reconoce a nivel mundial por constituirse como una actividad notable que se fundamenta en el esfuerzo cooperativo que el hombre desarrolla en las organizaciones públicas y privadas, en una sociedad caracterizada por una creciente complejidad en sus relaciones, y una mayor dinámica en los procesos de cambio, transformación y adecuaciones de las cuestiones administrativas que tienen que ver con el progresivo flujo de bienes, servicios, personas y dinero que se realizan al interior de cada país y a través de las fronteras nacionales. (Méndez Sánchez V M, Méndez Ortiz L, Cruz Landa A J 2018)

Sobre la base de las consideraciones anteriores el fenómeno de globalización en la últimas décadas ha permeado significativamente en la mayor parte de los países, entre los procesos, anteriormente expuesto se destaca la magnitud de su influencia, que, lo que a su vez ha derivado en una interdependencia económica, social, política y tecnológica principalmente, entre estos; así como a una situación de constante dinamismo y competitividad, que ha concebido la necesidad por parte de los dirigentes de las organizaciones, a entender las complejas cuestiones que éstas 
tienen que enfrentar, así como desarrollar sus capacidades para lograr el éxito a largo plazo. Si bien la tarea no sólo consiste en establecer estrategias a largo plazo y esperar a ver cómo funciona, ya que el ritmo del cambio se ha acelerado demasiado, de tal forma que hay que anticiparse a dichos cambios y mejorar continuamente las estrategias, aplicando mejoras en éstas en caso de ser necesario. (Méndez, Sánchez V M, Méndez Ortiz L, Cruz Landa AJ. 2018)

La administración en su situación actual, ha pasado por un proceso evolutivo en el que cada etapa de su historia, desde el surgimiento de la administración científica, se caracteriza por el desarrollo de los avances teóricos, herramientas y metodologías que las necesidades y problemáticas específicas de cada época han requerido. (Méndez Sánchez V M, Méndez Ortiz L, Cruz Landa A J 2018)

Gento (2000), define la función administrativa Cito (...) "como el cumplimiento cabal de todos y cada una de las funciones del proceso administrativo: planificar, coordinar, dirección y evaluar de manera eficaz y eficiente.” Esto deja claro, la coordinación de procesos de la administración, su intención de sistematizar acciones para hacer posible los procesos, dirigir la norma y racionalizar recursos en atención a un plan o proyecto.

El análisis del proceso administrativo se vuelve más complejo cuando los aplicamos en las organizaciones.

Abordan importantes investigadores que al referirse a las empresas estamos hablando de una organización; conjunto de personas que utilizando medios y recursos van a realizar una serie de actividades dirigidas a conseguir un objetivo común, por lo que se necesita profesionales con amplios conocimientos en el campo de la administración de organizaciones, que posean conocimientos conceptuales que enriquezcan la interpretación de los cambios que se producen en el entorno en el que se desenrolla la organización, como en su interior, y tener un conjunto de conocimientos técnicos en torno a herramientas complejas útiles para la gestión de organizaciones y con capacidades organizativas para dirigir organizaciones productivas, innovadoras y comprometidas con su medio.( Marco, F .2017). 
Es importante plantear que no todas organizaciones son empresas pero si todas las empresas son organizaciones con características comunes y diferentes.

De acuerdo a los razonamientos que han venido realizándose se conceptualiza la empresa como un sistema social en el que se integra un conjunto de personas y medios para el logro de sus objetivos. También se plantea que es la base del sistema económico, producen los bienes y servicios que son necesarios y a la vez proporcionan a los trabajadores el dinero preciso para comprarlos. Son entidades con personalidad jurídica propia. (La empresa y su entorno.2017.)

En ese mismo sentido se puede decir que la empresa es una entidad que integra un conjunto de elementos organizados y dirigidos para alcanzar una serie de objetivos mediante la realización de una determinada actividad económica.

Al analizar las diferentes definiciones de empresas observamos un denominador común que es la base de un sistema económico.

Según la Academia de Administración de los Estados Unidos la ciencia de la administración de empresas comúnmente incluye el estudio de cuatro áreas básicas: teoría organizacional (TO), administración estratégica (AE), comportamiento organizacional (CO), y dirección de recursos humanos (DRH). Las mismas están interconectadas y son interdependiente. (Fuentes Henríquez F 2005) 
Cuatros áreas básicas de la administración

\begin{tabular}{|c|l|}
\hline Teoría organizacional (TO), & Administración estratégica (AE), \\
\hline Comportamiento organizacional (CO) & Dirección de recursos humanos (DRH). \\
\hline Teórico & \\
\hline & Practico \\
\hline Énfasis & \\
\hline
\end{tabular}

En el cuadro se observa que la administración es una disciplina de alta complejidad, contiene el análisis teórico y práctico de los antecedentes y consecuencias de múltiples fenómenos organizacionales que suceden a nivel individual, grupal, organizacional, industrial y global (ej. Internacional). Ejemplificamos; comportamiento organizacional es el área de la administración que concentra sus esfuerzos en explicar múltiples fenómenos de la conducta individual y grupal (micro-administrativos) en una organización, la esencia de determinar los posibles antecedentes que explican el desempeño, satisfacción, motivación, compromiso y otras variables dependientes, a nivel individual, grupal y organizacional. (Fuentes Henríquez F 2005)

Investigaciones elaborados por expertos prestigiosos relacionada con la conducta organizacional tratan de explicar los fenómenos de la administración de recurso humanos (macroadministrativos). Por ejemplo, las diferentes teorías de la motivación han sido desarrolladas, en su mayoría, por estudiosos en la conducta organizacional; estas teorías son el componente central para diseñar e implementar diferentes herramientas y técnicas de recursos humanos, por 
ejemplo, sistema de compensación, evaluación de desempeño, etc. Igualmente, como el área del comportamiento organizacional alimenta a la administración de recursos humanos de los lineamientos teóricos, el área de teoría organizacional fomenta el área de administración estratégica para que esta defina y diseñe diferentes recomendaciones claves en la administración estratégica. Ejemplificamos; el área de teoría organizacional incluye el estudio de la teoría de agencia y de la teoría de los costos de transacción, ambas teorías son el eje central que explican la mayoría de las recomendaciones estratégicas que son realizadas por la administración estratégica en lo concerniente a la gestión de gobiernos corporativos, alianzas estratégicas, y estrategias internacionales. (Fuentes Henríquez F 2005)

Así de importante nos centraremos de forma breve pero muy estudiada algunos aspectos acerca del área de administración estratégica.

\section{Gerencia estratégica}

La globalización nos obliga a reconocer que el mercado en todo el planeta, se requiere diseñar planes de negocio que tomen en cuenta los recursos no importa su ubicación, clientes y proveedores de todos el orbe, con la finalidad de perfeccionar la acción empresarial. Es de importancia razonar que la economía de mercado es el campo natural donde las empresas se desempeñan. Frente a estos desafíos las organizaciones tienen el compromiso de ser lo

suficientemente flexibles para transformar toda la estructura de negocios, y así responder a cambios estratégicos y retos del mercado; Según se ha visto, para conseguir una posición de ventaja competitiva, es necesario examinar de manera crítica el entorno, con el objetivo de identificar oportunidades externas y crear capacidades internas; por lo que no pueden existir organizaciones estáticas deben de insertarse en un mundo dinámico, con altas exigencias que demandan una visión sistémica e integral.( González, J; Salazar, F; Ortiz, R; Verdugo, D . 2019)

Ahora las empresas buscan ser más eficaces, como resultado de la competencia que el mercado global establece entre ellas, por lo que un requisito organizacional es la implementación de herramientas y estrategias para alcanzar adecuadamente los objetivos trazados, dentro de su proceso de planeación estratégica. (González, J; Salazar, F; Ortiz, R; Verdugo, D. 2019) 
En este orden de ideas, se tiene que el objetivo de toda empresa es conseguir resultados favorables, lo cual se presenta con la diferenciación; ser diferente, en eso consiste la estrategia competitiva, esta solo se logra encontrando las ventajas competitivas (García, Prieto y García, 2016)

Bajo este contexto González, J et al (2019) conceptualiza la Gerencia Estratégica, como una herramienta para administrar y ordenar los cambios, donde se definen los objetivos de la organización y se establecen estrategias; así mismo, se reconoce la participación basada en el liderazgo y la toma de decisiones que correspondan a las demandas del ambiente inmediato y futuro.

Es de estimado valor plantear que el Pensamiento Estratégico añade valores, misión, visión y estrategia que tienden a ser elementos intuitivo, basado en los sentimientos, más que analítico, basados en información. Tener compromiso sobre estos elementos entre los miembros del equipo administrativo es una condición esencial para la planeación efectiva. Se infiere la importancia del pensamiento estratégico, como el germen para la toma de decisiones estratégicas, sin este fundamento las decisiones y acciones subsecuentes quizá sean fragmentadas e inconsistentes para el buen desempeño a largo plazo de la empresa (Chiavenato y Sapiro, 2011)

Con relación a la planificación estratégica, Friedmann (1997) citado por Alvarado, Yajaira, \& Paz, Dirmero. (2010) considera que puede ser concebida como una práctica profesional que busca la manera de concretar formas de conocimiento con formas de acción, debiendo operar los eventos reales de cada día, en vez del futuro, y que debe ser normativa, innovadora, política, transaccional y basada en el aprendizaje social. Igualmente, otros autores considera que la planificación estratégica es un proceso por el cual las organizaciones desarrollan instrucciones y actividades tendientes a determinar metas y objetivos a través del análisis del ambiente interno y del entorno, adoptan cursos de acción específicos y asignan recursos, para hacer posible la obtención de las metas de la organización.

De la misma forma Vivas (2000), expreso en su investigación, que para determinar el grado de presencia del pensamiento estratégico en las organizaciones hay que tener en cuenta los 
siguientes enfoque: actitud, cultura, herramienta, cualidad .Con relaciona a la actitud y cultura, en el pensamiento estratégico se define como un estado de conciencia, el cual permite hacer frente a situaciones difíciles del presente, con el fin de estar preparado ante el futuro. Este estado de conciencia de las organizaciones como actitud se manifiesta por la actitud gerencial estratégica, donde concentrarse en lo vital de la empresa o negocio, es hacer uso consciente de las herramientas gerenciales; definiendo el propósito y planteamiento estratégico en relación a la cultura, éste se manifiesta por el carácter proactivo de los individuos, visón global, sistema de valores reconocidos, promoción de la esencia del negocio, además de la adaptación a los cambios.

En resumen, es de apreciar que la planificación estratégica es un proceso que parte de la explicación y descripción de una realidad determinada por parte de diferentes actores sociales y que logra, a través de acciones de intervención, transformar la realidad y obtener la visión que estos deseen.

Hacemos referencias a las etapas de la planificación estratégica.

- Formulación de la estrategia: la formulación de la estrategia incluye la creación de una visión y misión, la identificación de las oportunidades y amenazas externas de una organización, la determinación de las fortalezas y debilidades internas, el establecimiento de objetivos a largo plazo, la creación de estrategias alternativas y la elección de estrategias específicas a seguir.( David, F. 2013).

- Ejecución de la estrategia: La implantación de la estrategia significa movilizar a los empleados y gerentes para poner en acción las estrategias formuladas. (García Guiliany, J E; Duran, S E; Cardeño Pórtela, E; Prieto Pulido, R; Garcia Cali, E; Paz Marcano, A. 2017)

- Evaluación de la estrategia: La evaluación de la estrategia es la etapa final de la dirección estratégica. Los gerentes necesitan saber cuándo ciertas estrategias no funcionan adecuadamente; y la evaluación de la estrategia es el principal medio para obtener 
información. (Garcia Guiliany, J E; Duran, S E; Cardeño Pórtela, E 3; Prieto Pulido, R ; Garcia Cali, E ; Paz Marcano, A 2017).

Dadas las condiciones que anteceden, obtener el ajuste estratégico garantizará la conciliación de la estrategia con el entorno, las características internas y la misión y objetivos elegidos. Por otro lado, el ajuste organizativo garantizará la adecuación de la organización en la que la estrategia va a ser implantada, lo que implica ajuste con el contexto y con la estrategia elegida.( Garcia Guiliany, J E ; Duran, S E ; Cardeño Pórtela, E 3; Prieto Pulido, R ; Garcia Cali, E ; Paz Marcano, A 2017)

Se considera que uno de los principales propósitos de la planificación estratégica, es que las organizaciones dispongan de un soporte que les permita asumir los procesos de cambio de manera eficiente, así como también que se encuentren dispuestas para gestionar transformaciones, obtener beneficios y permanecer en el mercado con niveles de competitividad, dado la alta movilización generada en el proceso de negociaciones de las empresas. Para ello, se requiere contar con aspectos claves, los cuales pasan a ser fundamentos sobre los cuales deba ajustar permanentemente, así como las interrelaciones entre estos aspectos (pensamiento sistémico). Por supuesto adecuándose a las características de cada empresa, tales como Pyme o multinacional, pública, mixta o privada. (Garcia Guiliany, J E; Duran, S E; Cardeño Pórtela, E 3; Prieto Pulido, R; Garcia Cali, E ; Paz Marcano, A 2017)

\section{Factores que influyen en el funcionamiento de una empresa}

(Febles Acosta, J., \& Oreja Rodríguez, J.R. (2008), (Helmut Sy Corvo. 2015 https://www.lifeder.com/factores-internos-externos-empresa/

Los factores internos y externos de una empresa son aquellos agentes que se encuentran dentro y fuera de una organización, que pueden generar un impacto positivo o negativo en la misma. Un concepto de negocio que se ve perfecto sobre el papel puede resultar imperfecto en el mundo real. 
A veces, la falla se debe al entorno interno: las finanzas, el personal o la maquinaria de la empresa. Otras veces es debido al entorno que rodea a la empresa. Saber cómo los factores internos y externos afectan a una empresa puede ayudar a que el negocio prospere.

Para su mayor comprensión listamos los factores internos y externos de una empresa.

Factores internos: Son todas las cosas que están dentro de la empresa y bajo su control, sin importar si son tangibles o intangibles; los mismos se agrupan en fortalezas y debilidades. Si un elemento trae efectos positivos a la empresa, se considera como fortaleza. Si un factor impide el desarrollo de la empresa, es una debilidad.

Está demostrado que estos factores determinan el avance de la organización tanto como una entidad organizacional autónoma como en respuesta a su entorno externo:

- Propietarios: grupo de personas que han invertido en la empresa y tienen los derechos de propiedad sobre la organización. Tienen el derecho de cambiar las políticas de la empresa en cualquier momento.

- Comunicación: El éxito de una organización esta extremadamente con el uso de las técnicas de comunicación. Las organizaciones con deficiencias comunicacionales a menudo tienen estructuras rígidas de liderazgo que destruyen la confianza.

- Estructura organizacional: La mayoría de las organizaciones tenían estructuras altamente jerárquicas, con muchos niveles gerenciales que definían a la organización de arriba a abajo. En la actualidad se ha entendido que las organizaciones con estructuras planas (con pocos niveles jerárquicos) superan a las que poseen estructuras altamente jerárquicas.

- Aprendizaje: Es una de las actividades humanas más fundamentales e influye, directa o indirectamente, en el éxito de toda empresa.

- Personal: Los empleados y también los sindicatos a los que se unen son muy importantes. $\mathrm{Si}$ se gestionan correctamente, pueden cambiar positivamente la política de la 
organización. Sin embargo, la mala gestión del personal podría conducir a una situación catastrófica para la empresa. El personal debe ser bueno haciendo su trabajo, los gerentes deben manejar su personal. Sin embargo, aunque todos sean capaces y talentosos, las políticas internas y los conflictos pueden arruinar a una buena empresa.

- Recursos de capital: Para que toda empresa lleve a cabo su plan el dinero es vital. Ninguna organización puede subsistir sin tener recursos de capital. Luego que una empresa tiene suficiente presupuesto, puede ejecutar fácilmente sus proyectos y crecer.

- Cultura organizacional: Es el comportamiento colectivo de los miembros de una organización y los valores, visiones, creencias, actitudes, prioridades y hábitos en los que se basan sus acciones. Juega un importante papel en la configuración del éxito empresarial, porque es determinante en lo bien que funcionará la organización. Toda organización tiene su propia cultura. Casi todo lo que afecte su capacidad para competir y responder con éxito a los cambios del entorno externo, así como a su propio éxito o fracaso, es consecuencia de esa cultura.

Factores externos: Elementos del entorno externo. La empresa no tiene control sobre cómo se formarán los mismos. Representan oportunidades o amenazas para la empresa.

El entorno externo se puede subdividir en dos capas:

- Entorno de tarea.

- Entorno general.

Entorno de tarea: Consiste en factores que afectan y se ven afectados directamente por las operaciones de la organización. Un gerente puede identificar estos factores como de interés específico, en lugar de tener que lidiar con las dimensiones más abstractas del entorno general:

- Competidores: Las políticas de una empresa con frecuencia están influenciadas por los competidores. Las empresas siempre intentan mantenerse en el mercado competitivo e ir más lejos que sus competidores. En la economía mundial actual, la competencia y los 
competidores han aumentado enormemente en todos los aspectos. El efecto positivo de esto es que los clientes siempre tienen opciones y la calidad general de los productos aumenta. La competencia puede hacer quebrar a una empresa: solo basta observar cuántas librerías se cayeron y cerraron compitiendo con Amazon.

- Clientes: El principal objetivo de toda empresa es la satisfacción del cliente. El cliente es quien paga el dinero por los productos o servicios de una organización. Son quienes otorgan los beneficios que las empresas están buscando. Se debe prestar atención por parte de los administrados a los clientes, porque la compra que ellos hagan es lo que mantiene viva y sólida a una empresa. El poder de los clientes dependerá de la agresividad de la competencia por el dinero de ellos, de la calidad que tengan los productos de la empresa y de la publicidad que haga que los clientes quieran comprar productos o servicios de esa empresa, entre otros elementos.

- Proveedores: Son los que suministran los materiales o servicios a una empresa. Relacionarse con los proveedores es una importante tarea gerencial. Una buena relación entre la empresa y los proveedores es trascendental para poder mantener un buen seguimiento sobre la calidad y costo de los materiales adquiridos.

Entorno general: Lo componen los factores que pueden tener un efecto directo inmediato sobre las operaciones de la empresa pero que, sin embargo, no influyen en sus actividades. Las dimensiones del entorno general son amplias y no específicas:

- Dimensión económica: Los factores económicos más importantes para la empresa son la inflación, las tasas de interés y el desempleo. Estos factores económicos siempre afectan la demanda de productos. Durante la inflación, la compañía tiene que pagar más por sus recursos y debe cubrir costos más altos, aumentando los precios de los productos. Cuando las tasas de interés son altas, los clientes están menos dispuestos a pedir dinero prestado y la empresa misma debe pagar más cuando pide prestado. Cuando el desempleo es alto, la empresa puede ser muy selectiva con respecto a quién contrata, pero el poder adquisitivo de los consumidores es bajo, por haber menos personas trabajando. 
- Dimensión tecnológica: Son los métodos disponibles para convertir los recursos en productos o servicios. Se debe tener cuidado con la dimensión tecnológica; la decisión de inversión debe ser precisa en las nuevas tecnologías y deben adaptarse a estas.

- Dimensión socio-cultural: Debe ser bien estudiada las costumbres, valores y características demográficas de la sociedad en la que opera la empresa.. Muestra el producto, servicios y estándares de conducta que la sociedad probablemente valorará y apreciará. El estándar de conducta del negocio varía de una cultura a otra y también el gusto y la necesidad de productos y servicios.

- Dimensión político-legal: Leyes comerciales, la relación entre el gobierno y las empresas y la situación político-legal general de un país. Las leyes comerciales establecen las obligaciones y los deberes de una empresa. Una adecuada relación entre el gobierno y las empresas es fundamental para la economía y, lo más importante, para la empresa. La situación general de implementación de la ley y la justicia en un país indica si hay una situación favorable para el éxito de la organización

- Dimensión internacional: Es grado en el que una empresa está implicada o afectada por los negocios en otros países. El concepto de sociedad global ha reunido a todas las naciones, y con la moderna red tecnológica comunicacional y de transporte, casi todas las partes del mundo están conectadas.

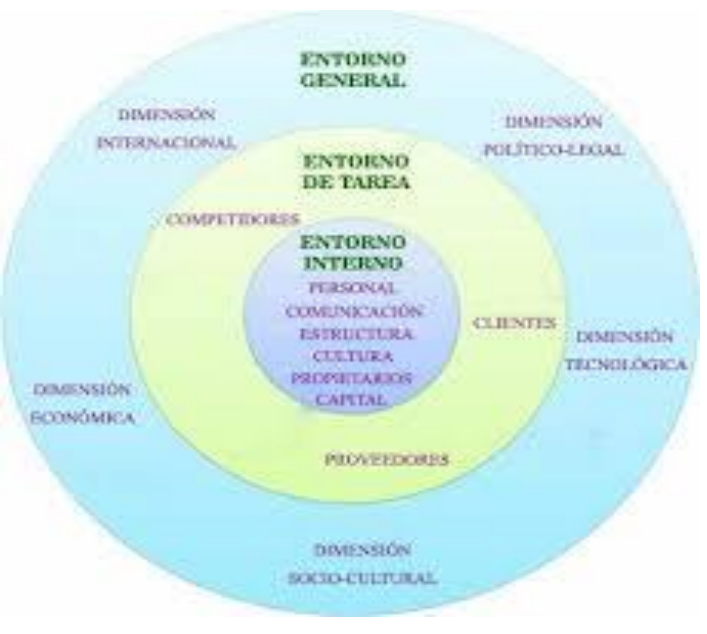




\section{Consideraciones finales}

El valor de una empresa está dado por el conjunto de elementos, materiales, inmateriales y humanos.

El recurso humano se convierte en el principal recurso competitivo de las empresas, convirtiéndose en el área con mayor valor estratégico.

El desempeño organizacional es el resultado de implementar y de enfocar el trabajo colaborativo de las nuevas formas de organización al detectar los cambios continuos que muestra el mercado y generar un clima de confianza que ayude y facilite el proceso de comunicación efectiva, para intercambiar puntos de vista y opiniones que busquen lograr desarrollar una innovación administrativa.

\section{Referencias}

1. Alvarado, Yajaira, \& Paz, Dirmero. (2010). Elementos del pensamiento estratégico en las empresas cooperativas. Revista de Ciencias Sociales, 16(3), 430-441. Recuperado, de http://ve.scielo.org/scielo.php?script=sci_arttext\&pid=S1315-

$95182010000300005 \& \operatorname{lng}=\mathrm{es} \& \ln g=\mathrm{es}$

2. Chiavenato, Idalberto y Sapiro, Arâo (2011). Planeación estratégica. Fundamentos y aplicaciones. Segunda Edición. McGraw-Hill Education. México.

3. David, F. (2013). Conceptos de administración estratégica, editorial Pearson, novena Edición

4. Febles Acosta, J., \& Oreja Rodríguez, J.R. (2008). FACTORES EXTERNOS E INTERNOS DETERMINANTES DE LA ORIENTACIÓN DE LA CULTURA ESTRATÉGICA DE LAS EMPRESAS. Investigaciones Europeas de Dirección y Economía de la Empresa, 14(1), Recuperado en: https://www.redalyc.org/articulo.oa?id=2741/274120247001 
5. Fuentes Henríquez F 2005 La ciencia de la administración de empresas: un análisis de sus componentes y de la Contribución de la revista economía y administración. Economía y administración o64,

6. Garcia Guiliany, J E ; Duran, S E ; Cardeño Pórtela, E 3; Prieto Pulido, R ; Garcia Cali, E ; Paz Marcano, A 2017. Proceso de planificación estratégica: Etapas ejecutadas en pequeñas y medianas empresas para optimizar la competitividad. Revista espacio .38(52). https://www.researchgate.net/publication/321059717_Proceso_de_planificacion_estrategi ca_Etapas_ejecutadas_en_pequenas_y_medianas_empresas_para_optimizar_la_competiti vidad.

7. González, Jorge; Salazar, Flor; Ortiz, Raúl; Verdugo, Darwin. 2019. Gerencia estratégica: herramienta para la toma de decisiones en las organizaciones. Telos, vol. 21, núm. 1 Recuperado de : https://www.redalyc.org/jatsRepo/993/99357718032/99357718032.pdf

8. García, J; Prieto, R, y García, E. (2016). Competitividad en pymes de confección textil.Una perspectiva desde las habilidades gerenciales de sus directivos. Libro factores explicativos de competitividad empresarial. México: Qartuppi editores.

9. Gento, S. (2000). Instituciones Educativas para la calidad total. Editorial Muralla. SA. Segunda edición.

10. Helmut Sy Corvo. (2015) Factores Internos y Externos de una Empresa https://www.lifeder.com/factores-internos-externos-empresa/

11. La empresa y su entorno.2017. https://www.edebe.com/educacion/documentos/830343-0529-830343_LA_EIE_CAS.pdf

12. Marco, F .2017 Gestión y Administración en las organizaciones: introducción . http://biblioteca.unaj.edu.ar/wp content/uploads/sites/8/2017/02/Introduccion-gestion-yadministracion-organizaciones.pdf 


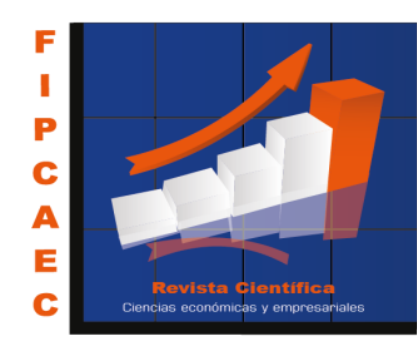

13. Méndez Sánchez V M, Méndez Ortiz L, Cruz Landa A J (2018): "La importancia de la administración en las organizaciones como forma para lograr competitividad", Revista Caribeña de Ciencias Sociales (enero 2018). En línea: http://www.eumed.net/rev/caribe/2018/01/administracion-organizaciones.html http://hdl.handle.net/20.500.11763/caribe1801administracion-organizaciones

14. Sánchez Jacas, Isolina. (2017). La planeación estratégica en el Sistema de Salud cubano. MEDISAN, 21(5), 635-641. Recuperado en 18 de octubre de 2019, de http://scielo.sld.cu/scielo.php?script=sci_arttext\&pid=S102930192017000500019\&lng=es\&tlng=es

15. Vivas, R. (2000). Gerencia y pensamiento estratégico. Material De Apoyo. Doctorado En Ciencias Gerenciales. Urbe. Maracaibo. Estado Zulia. Pp. 45-88.

16. Introducción a la administración. 2018. Semestre II. Facultad de ciencias administrativa y recurso humanos. Cuidad Universitaria. Lima Perú. https://www.usmp.edu.pe/estudiosgenerales/pdf/2018II/MANUALES/INTRODUCCION\%20A\%20LA\%20ADMINISTRACION.pdf

\section{References}

1. Alvarado, Yajaira, \& Paz, Dirmero. (2010). Elements of strategic thinking in cooperative companies. Journal of Social Sciences, 16 (3), 430-441. Recovered from http://ve.scielo.org/scielo.php?script=sci_arttext\&pid=S1315$95182010000300005 \& \operatorname{lng}=$ en\&tlng=en

2. Chiavenato, Idalberto and Sapiro, Arâo (2011). Strategic planning. Fundamentals and applications. Second edition. McGraw-Hill Education. Mexico.

3. David, F. (2013). Concepts of strategic management, Pearson editorial, 9th Edition 
4. Febles Acosta, J., \& Oreja Rodríguez, J.R. (2008). EXTERNAL AND INTERNAL FACTORS DETERMINING THE ORIENTATION OF STRATEGIC CULTURE OF COMPANIES. European Research on Business Management and Economics, 14 (1), Retrieved from: https://www.redalyc.org/articulo.oa?id=2741/274120247001

5. Fuentes Henríquez F 2005 The science of business administration: an analysis of its components and the contribution of the economy and administration magazine. Economics and administration o64,

6. Garcia Guiliany, J E; Duran, S E; Cardeño Pórtela, E 3; Prieto Polido, R; Garcia Cali, E; Paz Marcano, A 2017. Strategic planning process: Stages executed in small and medium enterprises to optimize competitiveness. Space magazine 38 (52). https://www.researchgate.net/publication/321059717_Process_of_planification_strategic_ Etapas_ejecutadas_en_pequenas_y_medianas_empresas_para_optimizar_la_competitivid ad.

7. González, Jorge; Salazar, Flor; Ortiz, Raúl; Executioner, Darwin. 2019. Strategic management: tool for decision making in organizations. Telos, vol. 21, no. 1 Recovered from: https://www.redalyc.org/jatsRepo/993/99357718032/99357718032.pdf

8. García, J; Prieto, R, and García, E. (2016). Competitiveness in textile manufacturing SMEs. A perspective from the managerial skills of their managers. Book explanatory factors of business competitiveness. Mexico: Qartuppi editors.

9. Gento, S. (2000). Educational institutions for total quality. Editorial Wall. SA. Second edition.

10. Helmut Sy Corvo. (2015) Internal and External Factors of a Company https://www.lifeder.com/factores-internos-externos-empresa/

11. The company and its environment. 2017. https://www.edebe.com/educacion/documentos/830343-0-529-830343_LA_EIE_CAS.pdf 
